\title{
The Influence of the Viscoelasticity on the Axisymmetric Wave Attenuation in a bi-Material Solid Cylinder
}

\author{
T. KOCAL ${ }^{a, *}$ AND S.D. AKBAROV ${ }^{b, c}$ \\ ${ }^{a}$ Yildiz Technical University, Department of Marine Engineering Operations, 34349 Istanbul, Turkey \\ ${ }^{b}$ Yildiz Technical University, Department of Mechanical Engineering, 34349 Istanbul, Turkey \\ ${ }^{c}$ Institute of Mathematics and Mechanics of the National Academy of Sciences of Azerbaijan, \\ 37041 Baku, Azerbaijan Technical University
}

\begin{abstract}
This paper studies the attenuation of the axisymmetric longitudinal waves propagating in a bi-material compound solid cylinder made of viscoelastic materials, the constitutive relations of which are described by utilizing the fractional exponential operators of Rabotnov. Rheological parameters are introduced and through these parameters the influence of the material viscosity on the attenuation coefficient of the propagating waves is investigated. $\mathrm{Nu}-$ merical results of the attenuation dispersion and the corresponding dispersion curves are presented and discussed. In particular, it is established that the dependence between the coefficient of attenuation and a certain rheological parameter can have a non monotonic character.
\end{abstract}

DOI: 10.12693/APhysPolA.134.135

PACS/topics: characteristic creep time, long-term elastic constants, wave attenuation, viscoelastic material, wave dispersion

\section{Introduction}

According to the review made in the papers by Akbarov et al. [1, 2] and Kocal and Akbarov [3] almost all investigations related to the wave dispersion in the elements of constructions made of viscoelastic materials have been made within the scope of the simplest Voigt model or within the scope of the hysteretic (nondispersive attenuation) model.

However, for more real and practical cases it is necessary to use more complicated and adequate viscoelastic models for investigations of the dispersion and attenuation of waves propagating in such structural elements as plates, cylinders, etc.

As an example for the mentioned complicated and real model, the model based on fractional exponential operators, developed by Rabotnov [4], can be taken. The attempts in this field have been made in the work of Akbarov et al. [1, 2] and Kocal and Akbarov [3] in the first two of which the dispersion of the axisymmetric longitudinal waves in bi-layered hollow and solid cylinders for the given attenuation case is investigated. However, in the third paper of the authors the attenuation of the axisymmetric longitudinal waves in the bi-layered hollow cylinder is investigated for given dispersion cases.

Taking into account the great significance of the results of Kocal and Akbarov [3], in the present paper the investigation is carried out for the solid cylinder made of bi-material, i.e. in the present paper the attenuation of the axisymmetric longitudinal

*corresponding author; e-mail: tkocal@yildiz.edu.tr waves in the bi-material compound solid cylinder is investigated for given possible wave dispersion cases.

\section{Formulation of the problem}

Consider the bi-material solid cylinder and assume that the radius of the cross-section circle of the inner cylinder is $R$ and the thickness of the outer covering hollow cylinder is $h$ (Fig. 1). The values related to the inner solid and external hollow cylinders will be denoted by the upper indices (2) and (1), respectively. Assume that the materials of the constituents are isotropic, homogeneous and hereditary-viscoelastic. We use the cylindrical system of coordinates $\operatorname{Or} \theta z$ to describe the positions of the points of the system under consideration. Moreover, we assume that the cylinders have infinite length in the direction of the $O z$ axis.

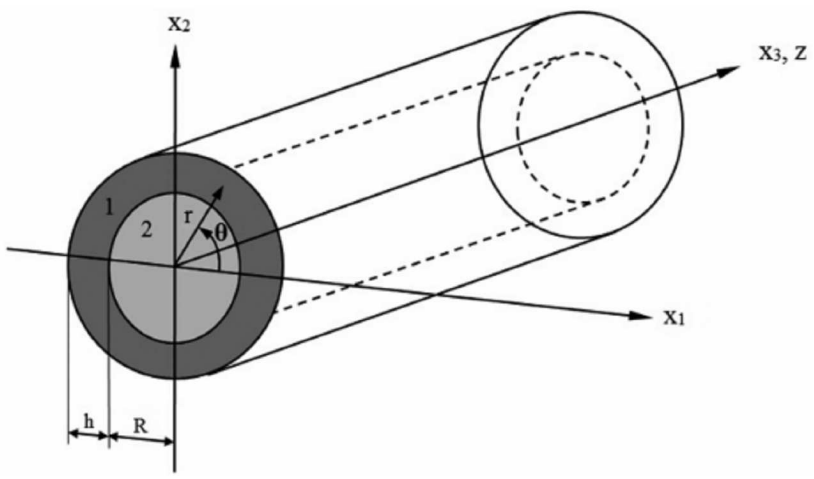

Fig. 1. The geometry of the compound cylinder.

Let us consider the axisymmetric wave attenuation along the $O z$ axis in the considered cylinder with the use of the following field equations and relations. 
Equations of motion:

$$
\begin{aligned}
& \frac{\partial T_{r r}^{(n)}}{\partial r}+\frac{\partial T_{r z}^{(n)}}{\partial z}+\frac{1}{r}\left(T_{r r}^{(n)}-T_{\theta \theta}^{(n)}\right)=\rho^{(n)} \frac{\partial^{2} u_{r}^{(n)}}{\partial t^{2}}, \\
& \frac{\partial T_{r z}^{(n)}}{\partial r}+\frac{\partial T_{z z}^{(n)}}{\partial z}+\frac{1}{r} T_{r z}^{(n)}=\rho^{(n)} \frac{\partial^{2} u_{z}^{(n)}}{\partial t^{2}} .
\end{aligned}
$$

Constitutive relations:

$$
\begin{aligned}
& T_{(i i)}^{(n)}=\lambda^{(n) *} \theta^{(n)}+2 \mu^{(n) *} \varepsilon_{(i i)}^{(n)}, \\
& (i i)=r r, z z, \theta \theta, \quad T_{r z}^{(n)}=2 \mu^{(n) *} \varepsilon_{r z}^{(n)}, \\
& \theta^{(n)}=\varepsilon_{r r}^{(n)}+\varepsilon_{\theta \theta}^{(n)}+\varepsilon_{z z}^{(n)},
\end{aligned}
$$

where $\lambda^{(n) *}$ and $\mu^{(n) *}$ are the following viscoelastic operators:

$$
\begin{aligned}
& \left\{\begin{array}{l}
\lambda^{(n) *} \\
\mu^{(n) *}
\end{array}\right\} \varphi(t)= \\
& \left\{\begin{array}{l}
\lambda_{0}^{(n)} \\
\mu_{0}^{(n)}
\end{array}\right\} \varphi(t)+\int_{0}^{t}\left\{\begin{array}{l}
\lambda_{1}^{(n)} \\
\mu_{1}^{(n)}
\end{array}\right\}(t-\tau) \varphi(\tau) \mathrm{d} \tau .
\end{aligned}
$$

Strain-displacement relations:

$$
\begin{array}{ll}
\varepsilon_{r r}^{(n)}=\frac{\partial u_{r}^{(n)}}{\partial r}, & \varepsilon_{r z}^{(n)}=\frac{1}{2}\left(\frac{\partial u_{r}^{(n)}}{\partial z}+\frac{\partial u_{z}^{(n)}}{\partial r}\right), \\
\varepsilon_{\theta \theta}^{(n)}=\frac{u_{r}^{(n)}}{r}, & \varepsilon_{z z}^{(n)}=\frac{\partial u_{z}^{(n)}}{\partial z} .
\end{array}
$$

In Eqs. (1-4) the conventional notation is used.

Consider also formulation of the boundary and contact conditions. According to Fig. 1 we can write the following boundary and contact conditions:

$$
\begin{aligned}
& \left.T_{(i j)}^{(2)}\right|_{r=R}=\left.T_{(i j)}^{(1)}\right|_{r=R},\left.\quad u_{(i)}^{(2)}\right|_{r=R}=\left.u_{(i)}^{(1)}\right|_{r=R}, \\
& \left.T_{(i j)}^{(1)}\right|_{r=R\left(1+h^{(1)} / R\right)}=0,
\end{aligned}
$$

$$
\text { where }(i j) \rightarrow r r ; r z, \quad(i) \rightarrow r ; z .
$$

This completes the formulation of the problem of the axisymmetric longitudinal wave dispersion in the bimaterial solid cylinder made of viscoelastic materials with arbitrary kernel functions $\lambda_{1}^{(n)}(t)$ and $\mu_{1}^{(n)}(t)$ which enter the constitutive relations $(2)$ and $(3)$.

The results obtained within the scope of the foregoing problem formulations allow one to determine the attenuation rule of the axisymmetric waves propagating in the compound solid cylinder made of viscoelastic materials.

These results are new not only in the sense of the new problem formulation, but also in the sense of using the fractional exponential operators for describing the viscoelastic properties of the materials. These operators allows to describe all physico-mechanical properties of the viscoelastic materials with high accuracy and in accordance with corresponding experimental data. At the same time, the results obtained within the scope of the foregoing formulation allow to control the wave attenuation through the rheological parameters of the materials of cylinders. Above-stated particularities of the considered problem determine the new physical insights on the wave propagation and its attenuation in the viscoelastic medium.

\section{Method of solution}

As we consider the time-harmonic wave propagation along the $O z$ axis, we can represent all sought values as $u_{(i)}(r, z, t)=v_{(i)}(r) \mathrm{e}^{(k z-\omega t)}, \varepsilon_{(i j)}(r, z, t)=$ $\gamma_{(i j)}(r) \mathrm{e}^{(k z-\omega t)}$. By carrying out the mathematical manipulations, made by authors in the foregoing papers, we obtain the following constitutive relations for the case under consideration.

$T_{(i i)}^{(n)}=\Lambda^{(n)} \vartheta^{(n)}(r) \mathrm{e}^{i(k z-\omega t)}+2 M^{(n)} \gamma_{(i i)}^{(n)}(r) \mathrm{e}^{i(k z-\omega t)}=$

$$
\sigma_{(i i)}^{(n)}(r) \mathrm{e}^{i(k z-\omega t)},
$$

$T_{r z}^{(n)}=2 M^{(n)} \gamma_{r z}^{(n)}(r) \mathrm{e}^{i(k z-\omega t)}=\sigma_{r z}^{(n)}(r) \mathrm{e}^{i(k z-\omega t)}$,

$\Lambda^{(n)}=\lambda_{0}^{(n)}+\lambda_{1 c}^{(n)}+i \lambda_{1 s}^{(n)}, \quad M^{(n)}=\mu_{0}^{(n)}+\mu_{1 c}^{(n)}+i \mu_{1 s}^{(n)}$.

The meaning of the notation used in (6) is given, for instance, in the paper by Akbarov et al. [1].

Thus, substituting the expression (6) into the equation of motion (1) and taking the relation (4) into consideration, we obtain the equations of motion, boundary and contact conditions in amplitude terms of the sought values.

For the solution of these equations, according to Guz [5], we employ the following representation for the displacement amplitudes:

$$
\begin{aligned}
v_{r}^{(n)} & =-i k \frac{\partial}{\partial r} \mathrm{X}^{(n)}, \\
v_{z}^{(n)} & =\frac{1}{m_{2}^{(n)}+m_{3}^{(n)}}\left(m_{1}^{(n)} \Delta_{1}-k^{2} m_{3}^{(n)}+\omega^{2} \rho^{(n)}\right) \mathrm{X}^{(n)}, \\
\Delta_{1} & =\frac{d^{2}}{d r^{2}}+\frac{1}{r} \frac{d}{d r}
\end{aligned}
$$

where $X^{(n)}$ satisfies equation

$$
\left[\left(\Delta_{1}-k^{2}\left(\zeta_{2}^{(n)}\right)^{2}\right)\left(\Delta_{1}-k^{2}\left(\zeta_{3}^{(n)}\right)^{2}\right)\right] X^{(n)}=0 .
$$

In (8) $\zeta_{2}^{(n)}$ and $\zeta_{3}^{(n)}$ are determined from the corresponding equations given, for instance, in the paper by Akbarov et al. [1].

Thus, the following expression for the function $X^{(n)}$ is obtained from Eq. (8)

$$
\begin{gathered}
X^{(n)}=A_{1}^{n} J_{0}\left(\zeta_{2}^{(n)} k r\right)+A_{2}^{n} J_{0}\left(\zeta_{3}^{(n)} k r\right) \\
+B_{1}^{n} Y_{0}\left(\zeta_{2}^{(n)} k r\right)+B_{2}^{n} Y_{0}\left(\zeta_{3}^{(n)} k r\right),
\end{gathered}
$$

where $J_{0}(x)$ and $Y_{0}(x)$ are Bessel functions of the first and second kinds with zeroth order, respectively.

Using expression (9) and the corresponding equations and relations given above we obtain the following dispersion equation:

$$
\operatorname{det}\left\|\beta_{n m}\right\|=0, \quad n ; m=1,2, \ldots, 6 .
$$

The explicit expressions of the components of the matrix $\left(\beta_{n m}\right)$ are given in the paper by Akbarov et al. [2]. 


\section{Numerical results and discussion}

According to the well-known physico-mechanical considerations, under time harmonic wave propagation in a viscoelastic material, it is necessary to assume that the wave number $k$ is a complex one and can be presented as $k=k_{1}+i k_{2}=k_{1}(1+i \beta), \beta=k_{2} / k_{1}$, where $k_{2}$ (or parameter $\beta$ ), i.e. the imaginary part of the wave number $k$, defines the attenuation of the wave amplitude under consideration and $\beta$ is called the coefficient of the attenuation.

In the present investigation we determine the values of the attenuation parameter $\beta$ from the dispersion equation (10) under possible selected values of the wave propagation velocity $c=\omega / k_{1}$ and the problem parameters $c / c_{20}^{(2)}, k_{1} R$ and $h^{(1)} / R$. Under this investigation the function $\mu_{1}(t)$ in (3), according to Rabotnov [4], is expressed through the function

$$
\begin{aligned}
& \Pi_{\alpha^{(n)}}^{(n)}\left(\varphi\left(\beta_{0}^{(n)}, \beta_{\infty}^{(n)}\right), t\right)= \\
& \quad t^{-\alpha^{(n)}} \sum_{p=0}^{\infty} \frac{\left(\varphi\left(\beta_{0}^{(n)}, \beta_{\infty}^{(n)}\right)^{(n) p} t^{\left.p\left(1-\alpha^{(n)}\right)\right)}\right.}{\Gamma\left((1+n)\left(1-\alpha^{(n)}\right)\right)}, \\
& 0 \leq \alpha^{(n)}<1 .
\end{aligned}
$$

In (11) $\Gamma(x)$ is the gamma function. Moreover, the constants $\alpha^{(n)}, \beta_{0}^{(n)}$ and $\beta_{\infty}^{(n)}$ in (11) are the rheological parameters of the material of the $n$-th layer.

As in the foregoing papers of the authors, we introduce the parameters

$$
\begin{aligned}
& d^{(n)}=\frac{\beta_{\infty}^{(n)}}{\beta_{0}^{(n)}} \\
& \text { and } Q^{(n)}=c_{20}^{(n)} /\left[R\left(\beta_{01}^{(n)}+\beta_{\infty}^{(n)}\right)^{\frac{1}{1-\alpha^{(n)}}}\right] .
\end{aligned}
$$

The mechanical meaning of the parameters in (12) is explained in the paper by Akbarov et al. [2], according to which, parameter $d^{(n)}$ characterizes the long-term values of the elastic constants of the $n$-th material. However, parameter $Q^{(n)}$ characterizes the creep time of these materials. An increase (a decrease) in the values of these parameters means a decrease (an increase) in the magnitude of the influence of the viscosity of the materials on the wave dispersion in the system under consideration.

Numerous numerical results and mechanical considerations show that the dispersion curves obtained for the corresponding purely elastic case with long-term values of the elastic constants (i.e. in the case where $d^{(1)}=d^{(2)}=0$ ) and the dispersion curves obtained for the corresponding purely elastic case with instantaneous values of the elastic constants (i.e. in the case where $\left.d^{(1)}=d^{(2)}=\infty\right)$, under satisfaction of certain conditions, can be taken as the lower and upper limit cases, respectively, for the dispersion curves obtained for the viscoelastic cases.

This statement allows us to select admissible dispersion curves and wave propagation velocity $c$ for the viscoelastic case and according to these curves (or velocity), to find the attenuation coefficient $\beta$ from the solution of the dispersion equation (10) for each fixed value of the dimensionless wavenumber $k_{1} R$.

For detailed illustration of the foregoing algorithm we consider the cases where $d^{(1)}=d^{(2)}=25$ and $d^{(1)}=d^{(2)}=35$, under $\nu_{0}^{(1)}=\nu_{0}^{(2)}=0.3\left(\right.$ where $\nu_{0}^{(k)}$ is the Poisson ratio of the $k$-th material), $h^{(1)} / R=0.1$, $\alpha^{(1)}=\alpha^{(2)}=0.5$ and $\mu_{0}^{(2)} / \mu_{0}^{(1)}=0.5$. Let us assume that $Q^{(1)}=Q^{(2)}$ and let us consider attenuation curves illustrated in Fig. 2 which are obtained in the cases where $d^{(1)}=d^{(2)}=25$ (Fig. 2a) and $d^{(1)}=d^{(2)}=35$ (Fig. 2b) by utilizing the algorithm described in the paper by Kocal and Akbarov [3]. Note that these attenuation curves correspond to the dispersion curves given in Fig. 3 which are also constructed for the cases where $d^{(1)}=d^{(2)}=25$ (Fig. 3a) and $d^{(1)}=d^{(2)}=35$ (Fig. 3b).
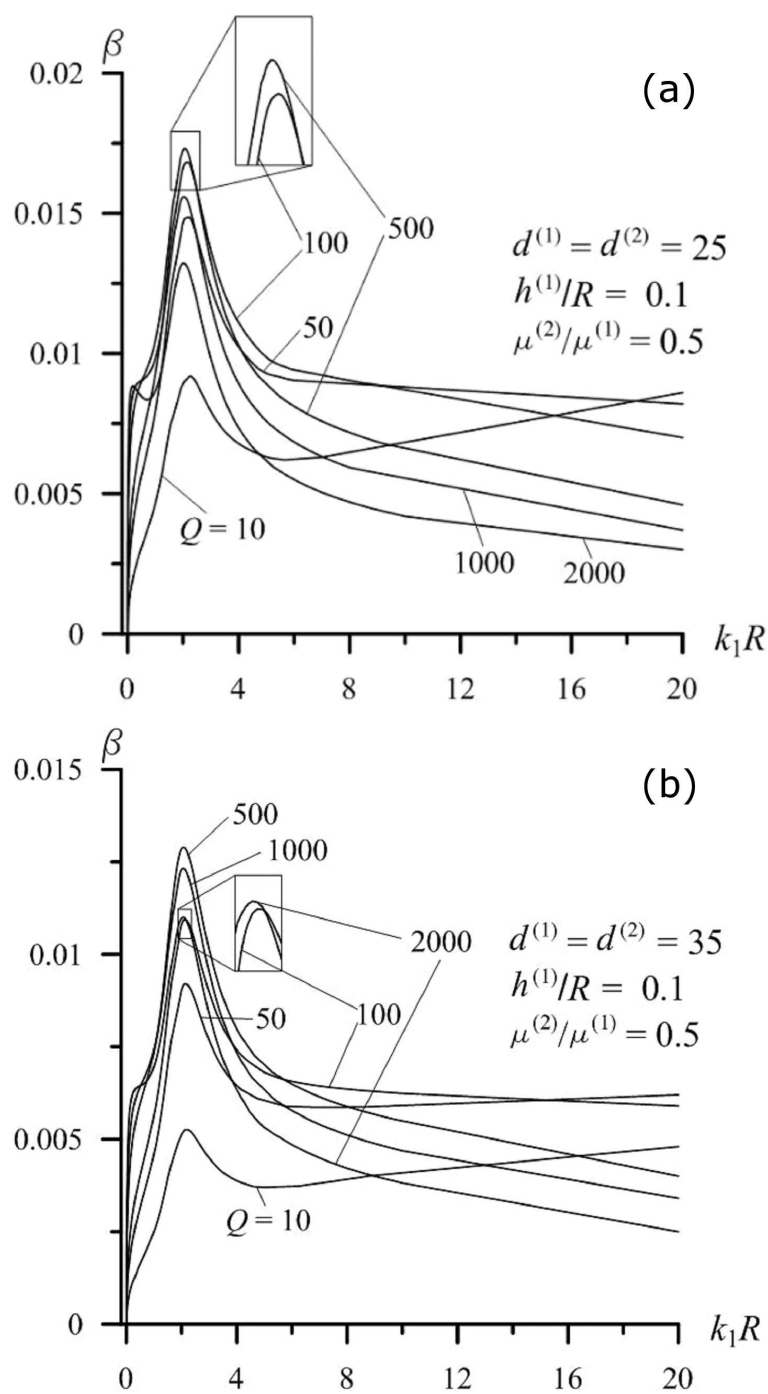

Fig. 2. Attenuation curves obtained in the cases where $d^{(1)}=d^{(2)}=25(\mathrm{a})$ and $d^{(1)}=d^{(2)}=35(\mathrm{~b})$ for the assumed dispersion curves indicated by numbers 1 and 2 in Fig. 3. 

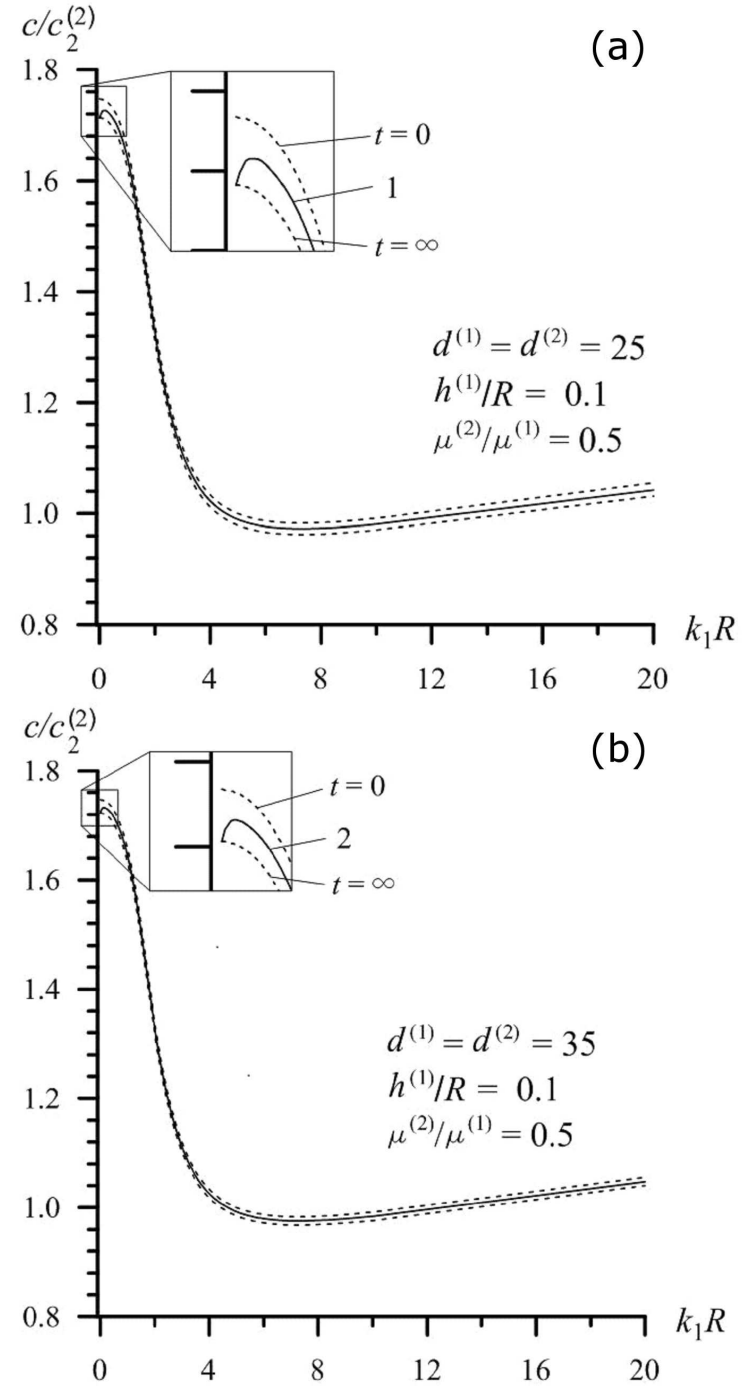

Fig. 3. Selected dispersion curves and dispersion curves obtained under instantaneous and long-term values of the elastic constants for the cases where $d^{(1)}=$ $d^{(2)}=25(\mathrm{a})$ and $d^{(1)}=d^{(2)}=35(\mathrm{~b})$.

It follows from Fig. 3 that the main attenuation of the waves under consideration takes place in the cases where $0<k_{1} R \leq 5$ and at a certain value of the wave-number $k_{1} R$ this attenuation has its maximum.

Moreover, the graphs given in Fig. 2 show that the dependence between the attenuation coefficient $\beta$ and the rheological parameter $Q$ has non monotonic character, i.e. transition below (above) a certain value of the $Q$ causes an increase (a decrease) in the values of the attenuation coefficient $\beta$.
Numerical results obtained for other problem parameters show that they in the quantitative sense are similar with those given here. Therefore we do not give these results here.

\section{Conclusions}

Thus, in the present paper the attenuation of the axisymmetric longitudinal waves, propagating in the bimaterial compound solid cylinder made of viscoelastic material, were calculated using the fractional exponential operators proposed by Rabotnov [4]. Numerical attenuation curves are presented and discussed and according to these curves the following conclusions are made:

- For the considered values of the problem parameters, the attenuation of the axisymmetric waves becomes more significant in the cases where $0<$ $k_{1} R<5$, i.e. the magnitude of the attenuation depends on the wavelength of the wave under consideration;

- Maximal values of the attenuation coefficient $\beta$ appear approximately at $k_{1} R \approx 2.0$, i.e. under $L \approx \pi R$, where $L$ is wavelength;

- There exists such a value of the rheological parameter $Q$ (we denote it by $Q^{*}$ ) for which $\max \{\beta\}$, i.e. the maximum of the attenuation coefficient with respect to the $k_{1} R$, has its maximum (we denote it by $\max \{\max \{\beta\}\}$. However, in the cases where $Q>Q^{*}$ (in the cases where $Q<Q^{*}$ ) an increase (a decrease) in the values of $Q$ causes a decrease in $\max \{\max \{\beta\}\}$;

- In the qualitative sense the obtained numerical results agree with the corresponding results obtained in the paper by Kocal and Akbarov [3].

\section{References}

[1] S. Akbarov, T. Kocal, T. Kepceler, Int. J. Solids Struct. 100-101, 195 (2016).

[2] S. Akbarov, T. Kocal, T. Kepceler, CMC: Computers Materials \& Continua 51(2), 105 (2016).

[3] T. Kocal, S. Akbarov, Structural Engineering and Mechanics 61(1), 143 (2017).

[4] Yu.N. Rabotnov, Elements of hereditary solid mechanics, Mir, Moscow 1980.

[5] A.N. Guz, Intern. Appl. Mech. 38, 23 (2002). 